\title{
Current and Future Use of Esophageal Capsule Endoscopy
}

\author{
Junseok Park' ${ }^{1}$ Young Kwan $\mathrm{Cho}^{2}$ and Ji Hyun Kim ${ }^{3}$ \\ Department of Internal Medicine, ${ }^{1}$ College of Medicine, Soonchunhyang University, Seoul, ${ }^{2}$ Eulji University School of Medicine, Seoul, ${ }^{3}$ Inje \\ University Busan Paik Hospital, Busan, Korea
}

Capsule endoscopy can be a diagnostic option for patients with esophageal diseases who cannot tolerate esophagogastroduodenoscopy. Functional modifications of the capsule allow for thorough examination of the esophagus. Esophageal capsule endoscopy has so far failed to show sufficient performance to justify the replacement of traditional endoscopy for the diagnosis of esophageal diseases because the esophagus has a short transit time and common pathologies appear near the esophagogastric junction. However, technological improvements are being introduced to overcome the limitations of capsule endoscopy, which is expected to become a good alternative to conventional endoscopy. Clin Endosc 2018;51:317-322

Key Words: Esophagus; Capsule endoscopy; Barrett’s esophagus; Esophageal varices

\section{INTRODUCTION}

Esophagogastroduodenoscopy (EGD) using a flexible endoscope is generally considered to be an optimal method for examining the esophagus. However, it carries disadvantages, such as discomfort and inconvenience, which may cause patients to decline necessary endoscopic evaluation. Simpler and less invasive modalities are needed as an alternative to traditional endoscopy. Video capsule endoscopy (VCE), widely used for investigating small bowel (SB) diseases, can be an acceptable option for the diagnosis of esophageal diseases. The esophagus normally has a short transit time and common pathologies tend to occur near the gastroesophageal junction. Therefore, VCE requires certain technical modifications for esophageal examination. In addition to technological improvements, tethering on a capsule can also improve VCE's

Received: June 8, 2018 Revised: July 17, 2018

Accepted: July 17, 2018

Correspondence: Ji Hyun Kim

Department of Internal Medicine, Inje University Busan Paik Hospital, 75 Bokji-ro, Busanjin-gu, Busan 47392, Korea

Tel: +82-51-890-6930, Fax: +82-51-892-0273, E-mail: zep2000@hanafos.com ORCID: https://orcid.org/0000-0003-0861-2792

*Junseok Park and Young Kwang Cho contributed to this paper equally.

(c) This is an Open Access article distributed under the terms of the Creative Commons Attribution Non-Commercial License (http://creativecommons.org/ licenses/by-nc/3.0) which permits unrestricted non-commercial use, distribution, and reproduction in any medium, provided the original work is properly cited. applicability to the esophagus. These adaptations allow VCE to be valuable in advanced diagnostic studies. This review will discuss recent developments and future prospects for esophageal VCE.

A capsule endoscopy system for the esophagus has been developed and is currently commercially available. The PillCam $\mathrm{ESO}^{\circledR}$ (Given Imaging Ltd., Yoqneam, Israel) was approved by the Food and Drug Administration in November 2004. Costing around $\$ 500$, the improved PillCam ESO2 ${ }^{\circledR}$ capsule has been available since 2007 (Fig. 1). The size, shape, and weight of the capsule endoscope are similar to those used in the SB (PillCam SB2) $(26 \times 11 \mathrm{~mm}$ and less than $3.0 \mathrm{~g})$. However, several functional modifications have been incorporated to optimize the capsule for esophageal evaluation. For example, the battery life is shortened to 20 minutes (rather than the 8-12 hours of SB capsules). In addition, cameras are placed on both

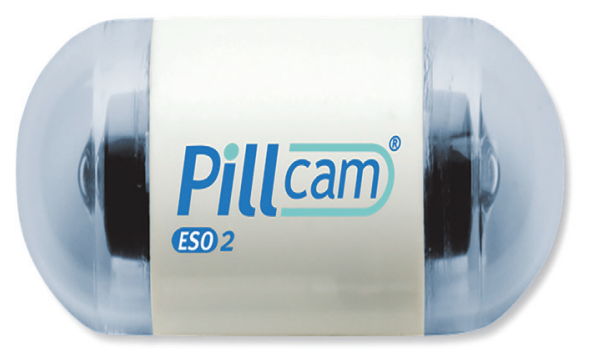

Fig. 1. PillCam ESO2 ${ }^{\circledR}$ (Given Imaging Ltd., Yoqneam, Israel) capsule. 
ends of the capsule and take images at 18 frames per second (fps; compared to 2-3 fps for SB capsules) with a wider angle $\left(169^{\circ}\right)$. A third-generation esophageal capsule endoscope, PillCam ESO3, with a wider angle of view $\left(174^{\circ}\right)$ and higher recording rate (35 frames/s), has already been introduced, although its practical advantage should be evaluated in a future study.

\section{PREPARATION AND PROCEDURES}

For an appropriately thorough examination, the patient should be fasting for over 2 hours. Generally, the patient drinks $100 \mathrm{~mL}$ of water while standing and then ingests the capsule in the supine position (Fig. 2). Additional 10-mL sips of water can be administered through a syringe or straw. A 5 -minute ingestion protocol is recommended. Two-minute recordings in the supine and $30^{\circ}$ inclined position are followed by a 1 -minute recording at $60^{\circ}$. A subsequent 15 -minute recording is acquired in the upright position. The images ob-

\section{Fasting for over 2 hours}

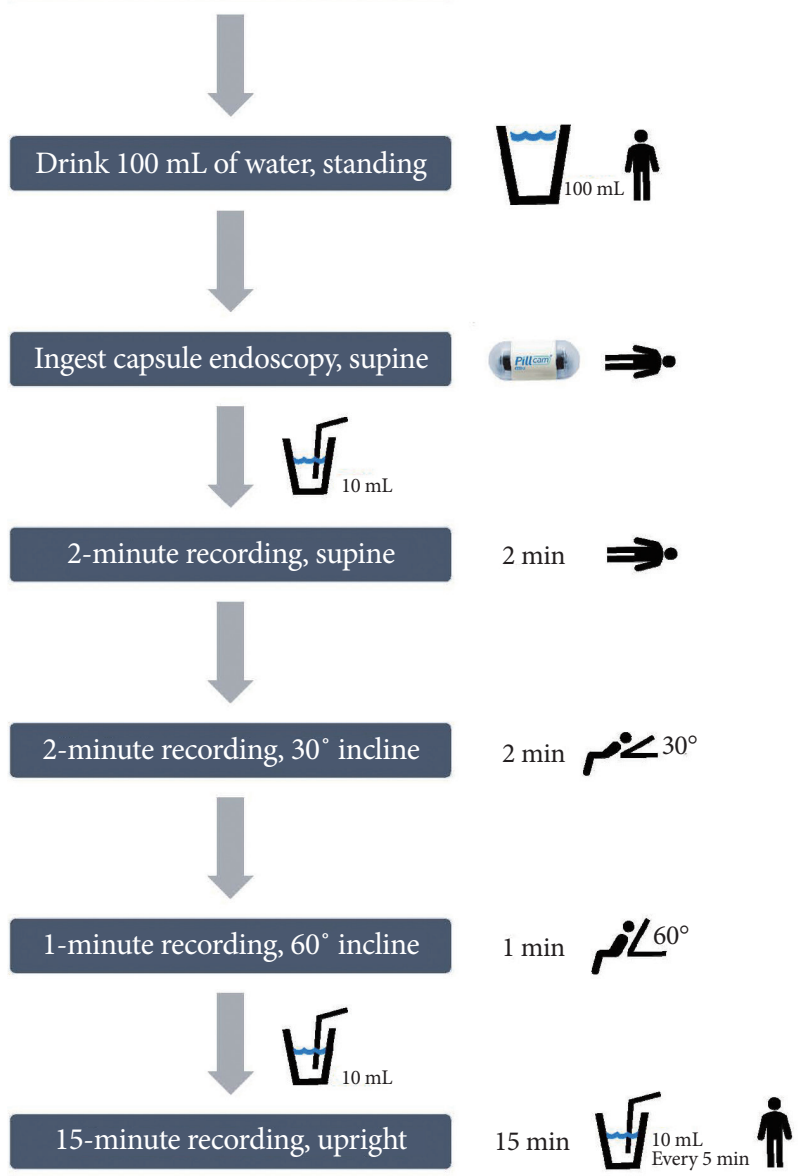

Fig. 2. Schematic process of esophageal capsule endoscopy. tained are transmitted via three thoracic sensors to the recorder.

\section{CURRENT EXPERIENCES WITH ESOPHAGEAL CAPSULE ENDOSCOPY}

Several studies have demonstrated the effectiveness of esophageal capsule endoscopy (ECE) through comparisons with EGD as the reference standard. Most studies were conducted on patients with Barrett's esophagus (BE) or esophageal varices (EV). As a minimally invasive diagnostic tool, ECE can be an option for screening patients who cannot tolerate EGD.

\section{ECE for gastroesophageal reflux disease and Bar- rett's esophagus}

Endoscopic evaluation is recommended for patients with gastroesophageal reflux disease (GERD) and its complications, especially $\mathrm{BE}$, which has malignant potential. ${ }^{1}$ Traditional endoscopy is limited by its cost, inconvenience, and the invasiveness of sedation. ${ }^{2,3}$ Several studies have been conducted to evaluate capsule endoscopy as a less invasive and more convenient endoscopic procedure for diagnosing GERD and BE.

A capsule endoscope especially designed for esophageal examination was introduced in 2004. ${ }^{4}$ The capsule was mechanically modified with two cameras located on both ends. It can obtain images at $4 \mathrm{fps}$ for approximately 20-30 minutes. Using the capsule endoscope, Eliakim et al. evaluated 17 patients with positive findings on EGD in the supine position. ${ }^{4}$ They achieved better detection rates, sensitivity, specificity, positive predictive values (PPV), and negative predictive values (NPV) (70.6\%, 100\%, 80\%, 92\%, and $100 \%$, respectively).

The increased image acquisition ability has improved the diagnostic yield of capsule endoscopy. In 2006, Koslowsky et al. investigated 2 types of capsules in 42 patients with GERD symptoms and 8 patients with confirmed BE. ${ }^{5}$ Compared with EGD, the 4-fps capsule showed sensitivity, specificity, PPV, and NPV rates of $81 \%, 61 \%, 74 \%$, and $79 \%$, respectively, while the 14 -fps capsule achieved rates of $100 \%$ ( $p<0.02$ ), $74 \%, 100 \%$, and $77 \%$, respectively. They concluded that the 14 -fps capsule has greater sensitivity and allows better visualization of the entire esophagus than the 4 -fps capsule.

The diagnostic accuracy of 14-fps capsule endoscopy was assessed in patients with $\mathrm{BE}$, esophagitis, and hiatal hernia. ${ }^{6}$ The authors compared the results of VCE with EGD in a blinded, prospective study. The results in the 94 patients with GERD symptoms and those undergoing BE surveillance showed a higher diagnostic accuracy for $\mathrm{BE}$ than for erosive esophagitis. The sensitivity, specificity, PPV, and NPV for BE in GERD patients were $67 \%, 87 \%, 60 \%$, and $90 \%$, respectively, while those for erosive esophagitis were $50 \%, 90 \%, 56 \%$, and 
$88 \%$, respectively. However, the authors concluded that the diagnostic rates for $\mathrm{BE}$ were not sufficiently accurate for VCE to replace EGD as the standard diagnostic modality.

Galmiche et al. investigated the diagnostic yield of ECE in a prospective multicenter trial of patients with chronic reflux symptoms. ${ }^{7}$ The study enrolled 77 patients, including 24 with esophagitis and 10 with esophageal metaplasia (ESEM), and validated the findings of ECE with EGD. The 14-fps ECE system showed sensitivity for esophagitis and ESEM of 79\% and $60 \%$, specificity of $94 \%$ and $100 \%$, PPV of $83 \%$ and $100 \%$, and NPV of $92 \%$ and $95 \%$, respectively. The authors concluded that ECE can be used for screening, but its exact role in detecting esophagitis and ESEM requires further investigation.

A meta-analysis was conducted to evaluate the diagnostic accuracy of ECE for BE in patients with GERD. ${ }^{8}$ Bhardwaj et al..$^{8}$ evaluated 618 patients from 9 studies (Table 1). ${ }^{5,7,9-13}$ The pooled sensitivity and specificity of ECE for the diagnosis of $\mathrm{BE}$ in all studies were $77 \%$ and $86 \%$, respectively; those of EGD, as the reference standard, were $78 \%$ and $90 \%$, respectively. The results adjusted for publication bias demonstrated that ECE has a moderate sensitivity and specificity for the diagnosis of BE in patients with GERD. However, the authors suggested that EGD remains the modality of choice for evaluation of suspected $\mathrm{BE}$.

\section{ECE for esophageal varices}

$\mathrm{EV}$ are highly prevalent in cirrhotic patients, and complications from bleeding are associated with a high rate of mortality. ${ }^{14}$ Endoscopic screening is recommended for patients with liver cirrhosis. ${ }^{15}$ These patients are at an inherently higher risk of complications during traditional endoscopy, in addition to the common cardiopulmonary complications related to sedation and analgesia. ${ }^{16,17}$ ECE was investigated as a less-invasive alternative to EGD for diagnosing EV.

Ramirez et al. reported the first study focusing on VCE for EV in 2005. ${ }^{18}$ They evaluated 30 cirrhotic patients with string-capsule endoscopy. The string-attached SB capsule en- doscope showed $96.7 \%$ accuracy in the diagnosis of $\mathrm{EV}$ and was preferred by the majority of patients (83.3\%).

The first 2 comparative studies were published in 2006, in which ECE showed a high diagnostic yield for EV in cirrhotic patients. Eisen et al. compared the results of the 14 -fps capsule endoscope with those of EGD in 32 patients. ${ }^{19}$ The reported sensitivity, specificity, positive likelihood ratio, and negative likelihood ratio were $100 \%, 89 \%, 9.1$, and 0.0 , respectively. Lapalus et al. presented the results of their comparison in 21 cirrhotic patients. ${ }^{20}$ The ECE system successfully detected the $\mathrm{EV}$ in $85 \%$ of the cases and indicated a need for primary prophylaxis in $100 \%$.

A large, multicenter prospective study of 120 patients was conducted and showed the sensitivity, specificity, NPV, and PPV of ECE for the detection of EV to be 77\%, 86\%, 69\%, and $90 \%$, respectively. ${ }^{21}$ The sensitivity, specificity, NPV, and PPV for indicating primary prophylaxis were $77,88,90$, and $75 \%$, respectively. The authors concluded that ECE is feasible, safe, well tolerated, and accurate for the diagnosis of EV. They also suggested that ECE could be an alternative to EGD in the evaluation of EV, for the screening of cirrhotic patients, and for indicating primary prophylactic treatment.

A study preforming both ECE and EGD in 50 cirrhotic patients reported that the accuracy of ECE in deciding the need for prophylaxis was $74 \%$, with a sensitivity of $63 \%$ and specificity of $82 \%{ }^{22}$ Inter-rater agreement was moderate (kappa $=0.56$ ). Agreement between EGD and ECE on grade of varices was also moderate (kappa $=0.53)$ and inter-rater reliability was good (kappa $=0.77$ ). In contrast to the former study, the authors concluded that ECE has a limited role in deciding which patients would need primary prophylaxis.

The results of a large, prospective, comparative study of 300 patients undergoing EV screening using ECE and EGD were published in $2015{ }^{23}$ The French cirrhotic patients were evaluated with ECE followed by EGD. The diagnostic indices of ECE for diagnosing and staging EV were: sensitivity, $76 \%$ and 64\%; specificity, 91\% and 93\%; PPV, 88\% and 88\%; and NPV,

Table 1. Pooled Sensitivity and Specificity of Esophageal Capsule Endoscopy in Diagnosing Barrett's Esophagus

\begin{tabular}{lcccccc}
\hline Author & Year & No. of patients & Reference standard & Capsule type & Sensitivity (\%) & Specificity (\%) \\
\hline Eliakim et al. & & 2005 & 106 & EGD & PillCam ESO & 97 \\
Koslowsky et al. $^{5}$ & 2006 & 50 & EGD & PillCam ESO & $75-100$ & $100-100$ \\
Lin et al. $^{10}$ & 2007 & 90 & Histology & PillCam ESO & 67 & 84 \\
Galmiche et al. $^{7}$ & 2008 & 77 & EGD & PillCam ESO & 60 & 100 \\
Sharma et al. $^{11}$ & 2006 & 94 & EGD & PillCam ESO & 77 & 95 \\
Gralnek et al. $^{12}$ & 2008 & 28 & EGD & PillCam ESO2 & 100 & 74 \\
Ramirez et al. $^{13}$ & 2008 & 100 & EGD & Tethered PillCam SB & 78 & 89 \\
\hline
\end{tabular}

EGD, esophagogastroduodenoscopy; SB, small bowel. 
$81 \%$ and $78 \%$, respectively. Patient satisfaction was assessed using the visual analog scale, and the ECE scores were significantly higher ( $87 \pm 22$ vs. $58 \pm 5, p<0.0001)$. The authors suggested that ECE specificity and PPV results indicated it can play a role in patients with contraindications for EGD or those who refuse it.

Recently, McCarty et al. evaluated ECE for the diagnosis and grading of medium to large EV in patients with portal hypertension in a systematic review and meta-analysis. ${ }^{24}$ The authors collected 17 studies performed between 2005 and 2015 for a total patient pool of 1,328..$^{18-21,23,25-31}$ The diagnostic accuracy of ECE in the diagnosis of EV was 90\% (95\% confidence interval [CI], 0.88-0.93). The diagnostic pooled sensitivity and specificity were $83 \%$ (95\% CI, $0.76-0.89)$ and $85 \%$ (95\% CI, 0.75-0.91), respectively (Table 2). The diagnostic accuracy of ECE in the grading of varices was 92\% (95\% CI, 0.90-0.94) and the pooled sensitivity and specificity were $72 \%$ (95\% CI, 0.54-0.85) and 91\% (95\% CI, 0.86-0.94), respectively. Based on these results, ECE was not considered capable of replacing EGD for diagnosing EV. However, it may be used in patients who are contraindicated for EGD or those who refuse it.

The diagnostic yield of ECE has proven insufficient for it to replace EGD in the evaluation of esophageal diseases. How- ever, ECE has continuously evolved with developments in technology, and can be a reasonable option for patients who are reluctant to undergo traditional endoscopy.

\section{ADDITIONAL APPLICATIONS}

Chen et al. introduced string ECE with real-time viewing. ${ }^{32}$ They evaluated the cotton string-attached PillCam ESO capsule in 30 healthy volunteers with a real-time monitoring program. Compared with conventional ECE, the string-attached ECE showed improved Z-line visualization (at least two-quadrant visualization, $100.0 \%$ vs. $80.0 \%, p<0.001$; at least three-quadrant visualization, $90.0 \%$ vs. $36.7 \%, p<0.001){ }^{32}$

A novel optomechanically-engineered device that can capture cross-sectional microscopic images has also been introduced. $^{33}$ This swallowable tethered capsule has been named optical coherence tomography endomicroscopy. Gora et al. presented their in vivo experience using tethered capsule endomicroscopy for BE screening. ${ }^{34,35}$

Table 2. Pooled Sensitivity and Specificity of Esophageal Capsule Endoscopy in Diagnosing Esophageal Varices

\begin{tabular}{lcccccc}
\hline Author & Year & No. of patients & Reference standard & Capsule type & Sensitivity (\%) & Specificity (\%) \\
\hline Ramirez et al. $^{18}$ & 2005 & 30 & EGD & Tethered M2A & 96 & 100 \\
Eisen et al. $^{19}$ & 2006 & 32 & EGD & PillCam ESO & 100 & 89 \\
Lapalus et al. $^{20}$ & 2006 & 21 & EGD & PillCam ESO & 68 & 100 \\
de Franchis et al. $^{27}$ & 2008 & 288 & EGD & PillCam ESO & 84 & 88 \\
Pena et al. $^{29}$ & 2008 & 20 & EGD & PillCam ESO & 68 & 100 \\
Lapalus et al. $^{21}$ & 2009 & 120 & EGD & PillCam ESO & 77 & 86 \\
Schreibman et al. $^{30}$ & 2011 & 37 & EGD & PillCam ESO & 65 & 67 \\
Ishiguro et al. $^{28}$ & 2012 & 29 & EGD & PillCam ESO & 95 & 78 \\
Chavalitdhamrong et al. $^{25}$ & 2012 & 65 & EGD & PillCam ESO & 83 \\
Stipho et al. $^{31}$ & 2012 & 100 & EGD & Tethered PillCam SB & 84 & 47 \\
Aoyama et al. $^{26}$ & 2014 & 119 & EGD & PillCam SB/SB2 & 72 & 72 \\
Sacher-Huvelin et al. $^{23}$ & 2015 & 330 & EGD & PillCam ESO2 & 76 \\
\hline
\end{tabular}

EGD, esophagogastroduodenoscopy; SB, small bowel.

Table 3. Comparisons of Procedural Characteristics between Esophageal Capsule Endoscopy and Traditional Esophagogastroduodenoscopy

\begin{tabular}{lcc}
\hline & Capsule endoscopy & Esophagogastroduodenoscopy \\
\hline Histological tissue sampling & Impracticable & Practicable \\
Luminal insufflation & Impracticable & Practicable \\
Locomotion control & Impracticable & Practicable \\
Therapeutic procedure & Impracticable & Practicable \\
Conscious sedation & Not necessary & Usually required \\
\hline
\end{tabular}




\section{LIMITATIONS AND FUTURE PROSPECTS OF ECE}

In addition to the relatively low diagnostic accuracy, ECE has other limitations to its ability to diagnose esophageal diseases. For instance, it lacks the ability to obtain histological samples or perform therapeutic procedures, both of which are required during the diagnosis and surveillance of $\mathrm{BE}$ (Table 3 ). Air insufflation is not possible using capsule endoscopy and proper expansion of the esophagus is necessary to accurately diagnose and grade EV. Moreover, endoscopic treatment, including band ligation and chemical sclerotherapy, cannot be performed with ECE.

Because of these limitations, ECE is not recommended as a first-line diagnostic tool. However, continued technical improvements are being introduced to advance the diagnostic yield of capsule endoscopy. Remote magnetic control systems allow it to overcome the rapid transit time of the esophagus. ${ }^{36}$ Modification of the light source with a flexible spectral imaging color enhancement system has been adopted to help diagnose esophageal malignancy. ${ }^{37}$ Recent ex vivo research has demonstrated that targeted biopsy using magnetic capsule endoscopy and a self-folding microgripper is possible. ${ }^{38}$

\section{CONCLUSIONS}

Capsule endoscopy has been proven to be a feasible and safe diagnostic option for patients with esophageal diseases. Several studies have reported promising results in screening for $\mathrm{BE}$ and $\mathrm{EV}$. While the results do not support the replacement of EGD with capsule endoscopy as the gold standard diagnostic modality for esophageal diseases, the recently developed PillCam ESO3 may be used in patients who refuse or are contraindicated for EGD. Capsule endoscopy should have more diagnostic abilities to overcome the physiological and anatomical characteristics of the esophagus. Technological advances are improving the value of capsule endoscopy in conjunction with locomotion control and tissue sampling, and will provide the benefits of capsule endoscopy in the diagnosis of esophageal disease.

\section{Conflicts of Interest}

The authors have no financial conflicts of interest.

\section{REFERENCES}

1. Wang KK, Sampliner RE. Updated guidelines 2008 for the diagnosis, surveillance and therapy of Barrett's esophagus. Am J Gastroenterol
2008;103:788-797.

2. Lapalus MG, Saurin JC. [Complications of gastrointestinal endoscopy: gastroscopy and colonoscopy]. Gastroenterol Clin Biol 2003;27:909-921.

3. Daneshmend TK, Bell GD, Logan RF. Sedation for upper gastrointestinal endoscopy: results of a nationwide survey. Gut 1991;32:12-15.

4. Eliakim R, Yassin K, Shlomi I, Suissa A, Eisen GM. A novel diagnostic tool for detecting oesophageal pathology: the PillCam oesophageal video capsule. Aliment Pharmacol Ther 2004;20:1083-1089.

5. Koslowsky B, Jacob H, Eliakim R, Adler SN. PillCam ESO in esophageal studies: improved diagnostic yield of 14 frames per second (fps) compared with $4 \mathrm{fps}$. Endoscopy 2006;38:27-30.

6. Sharma P, Wani S, Rastogi A, et al. The diagnostic accuracy of esophageal capsule endoscopy in patients with gastroesophageal reflux disease and Barrett's esophagus: a blinded, prospective study. Am J Gastroenterol 2008;103:525-532.

7. Galmiche JP, Sacher-Huvelin S, Coron E, et al. Screening for esophagitis and Barrett's esophagus with wireless esophageal capsule endoscopy: a multicenter prospective trial in patients with reflux symptoms. Am J Gastroenterol 2008;103:538-545.

8. Bhardwaj A, Hollenbeak CS, Pooran N, Mathew A. A meta-analysis of the diagnostic accuracy of esophageal capsule endoscopy for Barrett's esophagus in patients with gastroesophageal reflux disease. Am J Gastroenterol 2009;104:1533-1539.

9. Eliakim R, Sharma VK, Yassin K, et al. A prospective study of the diagnostic accuracy of PillCam ESO esophageal capsule endoscopy versus conventional upper endoscopy in patients with chronic gastroesophageal reflux diseases. J Clin Gastroenterol 2005;39:572-578.

10. Lin OS, Schembre DB, Mergener K, et al. Blinded comparison of esophageal capsule endoscopy versus conventional endoscopy for a diagnosis of Barrett's esophagus in patients with chronic gastroesophageal reflux. Gastrointest Endosc 2007;65:577-583.

11. Sharma P, Dent J, Armstrong D, et al. The development and validation of an endoscopic grading system for Barrett's esophagus: the Prague C \& M criteria. Gastroenterology 2006;131:1392-1399.

12. Gralnek IM, Adler SN, Yassin K, Koslowsky B, Metzger Y, Eliakim R. Detecting esophageal disease with second-generation capsule endoscopy: initial evaluation of the PillCam ESO 2. Endoscopy 2008;40:275-279.

13. Ramirez FC, Akins R, Shaukat M. Screening of Barrett's esophagus with string-capsule endoscopy: a prospective blinded study of 100 consecutive patients using histology as the criterion standard. Gastrointest Endosc 2008;68:25-31.

14. Lay CS, Tsai YT, Teg CY, et al. Endoscopic variceal ligation in prophylaxis of first variceal bleeding in cirrhotic patients with high-risk esophageal varices. Hepatology 1997;25:1346-1350.

15. Garcia-Tsao G, Sanyal AJ, Grace ND, Carey W. Prevention and management of gastroesophageal varices and variceal hemorrhage in cirrhosis. Hepatology 2007;46:922-938.

16. Benjamin SB. Complications of conscious sedation. Gastrointest Endosc Clin N Am 1996;6:277-286.

17. McGuire BM. Safety of endoscopy in patients with end-stage liver disease. Gastrointest Endosc Clin N Am 2001;11:111-130.

18. Ramirez FC, Hakim S, Tharalson EM, Shaukat MS, Akins R. Feasibility and safety of string wireless capsule endoscopy in the diagnosis of esophageal varices. Am J Gastroenterol 2005;100:1065-1071.

19. Eisen GM, Eliakim R, Zaman A, et al. The accuracy of PillCam ESO capsule endoscopy versus conventional upper endoscopy for the diagnosis of esophageal varices: a prospective three-center pilot study. Endoscopy 2006;38:31-35.

20. Lapalus MG, Dumortier J, Fumex F, et al. Esophageal capsule endoscopy versus esophagogastroduodenoscopy for evaluating portal hypertension: a prospective comparative study of performance and tolerance. Endoscopy 2006;38:36-41.

21. Lapalus MG, Ben Soussan E, Gaudric M, et al. Esophageal capsule endoscopy vs. EGD for the evaluation of portal hypertension: a French prospective multicenter comparative study. Am J Gastroenterol 
2009;104:1112-1118.

22. Frenette CT, Kuldau JG, Hillebrand DJ, Lane J, Pockros PJ. Comparison of esophageal capsule endoscopy and esophagogastroduodenoscopy for diagnosis of esophageal varices. World J Gastroenterol 2008;14:44804485.

23. Sacher-Huvelin S, Calès P, Bureau C, et al. Screening of esophageal varices by esophageal capsule endoscopy: results of a French multicenter prospective study. Endoscopy 2015;47:486-492.

24. McCarty TR, Afinogenova Y, Njei B. Use of wireless capsule endoscopy for the diagnosis and grading of esophageal varices in patients with portal hypertension: a systematic review and meta-analysis. J Clin Gastroenterol 2017;51:174-182

25. Chavalitdhamrong D, Jensen DM, Singh B, et al. Capsule endoscopy is not as accurate as esophagogastroduodenoscopy in screening cirrhotic patients for varices. Clin Gastroenterol Hepatol 2012;10:254-258.el.

26. Aoyama T, Oka S, Aikata $\mathrm{H}$, et al. Is small-bowel capsule endoscopy effective for diagnosis of esophagogastric lesions related to portal hypertension? J Gastroenterol Hepatol 2014;29:511-516.

27. de Franchis R, Eisen GM, Laine L, et al. Esophageal capsule endoscopy for screening and surveillance of esophageal varices in patients with portal hypertension. Hepatology 2008;47:1595-1603.

28. Ishiguro H, Saito S, Imazu H, Aihara H, Kato T, Tajiri H. Esophageal capsule endoscopy for screening esophageal varices among Japanese patients with liver cirrhosis. Gastroenterol Res Pract 2012;2012:946169.

29. Pena LR, Cox T, Koch AG, Bosch A. Study comparing oesophageal capsule endoscopy versus EGD in the detection of varices. Dig Liver Dis 2008;40:216-223.

30. Schreibman I, Meitz K, Kunselman AR, Downey M, Le T, Riley T. Defining the threshold: new data on the ability of capsule endoscopy to discriminate the size of esophageal varices. Dig Dis Sci 2011;56:220-226.

31. Stipho S, Tharalson E, Hakim S, Akins R, Shaukat M, Ramirez FC. String capsule endoscopy for screening and surveillance of esophageal varices in patients with cirrhosis. J Interv Gastroenterol 2012;2:54-60.

32. Chen WS, Zhu LH, Li DZ, Chen L, Wu YL, Wang W. String esophageal capsule endoscopy with real-time viewing improves visualization of the distal esophageal Z-line: a prospective, comparative study. Eur J Gastroenterol Hepatol 2014;26:309-312.

33. Gora MJ, Sauk JS, Carruth RW, et al. Tethered capsule endomicroscopy enables less invasive imaging of gastrointestinal tract microstructure. Nat Med 2013;19:238-240.

34. Ughi GJ, Gora MJ, Swager AF, et al. Automated segmentation and characterization of esophageal wall in vivo by tethered capsule optical coherence tomography endomicroscopy. Biomed Opt Express 2016;7:409419.

35. Gora MJ, Simmons LH, Quénéhervé L, et al. Tethered capsule endomicroscopy: from bench to bedside at a primary care practice. J Biomed Opt 2016;21:104001.

36. Keller J, Fibbe C, Volke F, et al. Remote magnetic control of a wireless capsule endoscope in the esophagus is safe and feasible: results of a randomized, clinical trial in healthy volunteers. Gastrointest Endosc 2010;72:941-946.

37. Oka A, Amano Y, Kusunoki R, et al. Superficial esophageal cancer observed with the PillCam ESO 2 in combination with the flexible spectral imaging color enhancement system. Dig Endosc 2011;23:195-196.

38. Yim S, Gultepe E, Gracias DH, Sitti M. Biopsy using a magnetic capsule endoscope carrying, releasing, and retrieving untethered microgrippers. IEEE Trans Biomed Eng 2014;61:513-521. 